\title{
On Some Properties of SEP Effective CMEs
}

\author{
Nishant Mittal, ${ }^{1,2}$ Anshu Gupta, ${ }^{1}$ P. S. Negi, ${ }^{1}$ and Udit Narain ${ }^{1,3}$ \\ ${ }^{1}$ Astrophysics Research Group, Department of Physics, Meerut College, Meerut 250001, India \\ ${ }^{2}$ Department of Physics, Sunder Deep Group of Institutions, Ghaziabad 201001, India \\ ${ }^{3}$ Department of Physics, FIT Engineering College, Mawana Road, Meerut 250001, India
}

Correspondence should be addressed to Nishant Mittal, nishantphysics@gmail.com

Received 2 November 2011; Accepted 27 November 2011

Academic Editor: M. Richmond

Copyright (C) 2011 Nishant Mittal et al. This is an open access article distributed under the Creative Commons Attribution License, which permits unrestricted use, distribution, and reproduction in any medium, provided the original work is properly cited.

Solar energetic particles (SEP) are believed to originate from two different sources, solar flares and coronal mass ejections (CMEs). The large SEP events constitute a serious radiation hazard. The high-energy solar proton data obtained from NOAA Space Environment Services Center are used to investigate some statistical properties such as speed, apparent width, acceleration, latitude, mass, kinetic energy, and occurrence rate of SEP effective CMEs observed during the period 1997-2006 covering the solar cycle 23. It is found that SEP effective CMEs are nearly halo in nature, and they follow solar cycle variation. Such CMEs have higher speeds than those of non-SEP effective CMEs.

\section{Introduction}

The solar cycle and activity phenomena are some of the interesting physical processes that are least understood. Since the discovery of sunspots, their origin and formation still remains a mystery. In a similar way, physics of the recently discovered (compared to dates of sunspots' discovery) solar activity phenomenon, namely, the solar energetic particles (SEPs) is yet to be understood.

The Sun generates transient fluxes of nonthermal particles in interplanetary space, ranging from suprathermal to relativistic energies. In particular, solar energetic protons (SEPs) have been routinely detected by satellites since the descending phase of the 19th cycle and, before that time, only very major proton events were identified at the Earth (ground level enhancements). High-energy particles from the Sun were first observed [1] as sudden increases in intensity in ground-level ion chambers during the large solar events of February and March 1942. They come from solar flares in the low corona, from shock waves driven outward by coronal mass ejections (CMEs), from planetary magnetospheres, and from bow shocks [2]. Since this was long before the discovery of CMEs, it was natural to assume that the energetic particles came from the solar flares that often accompany large CMEs. McCracken et al. [3] showed that, unlike sunspots, SEP events follow the $\sim 80-100$-year
Gleissberg cycle rather faithfully. The largest SEP event in the last 400 years appears to be related to the flare observed by Carrington in 1859 [4], but the probability of SEP events with such large fluencies falls off sharply.

The high-energy protons from SEP events present hazards to space systems; in particular damaging science instruments/electronics or to astronauts. A reliable estimate of the high-energy particle environment should assure the mission success. Without it, system survivability is often ensured by setting grossly overconservative mission requirements, resulting in high mission costs, weight, and physically large systems. The generation of SEP events on the Sun and, the occurrence of high-energy particles on the Earth orbit is, on one hand, the probabilistic phenomenon, and, on the other hand, it is related to changing of the solar activity (SA).

Reames [2] in his review states that "planets, and their interaction with the interplanetary medium, can be a rich source of energetic particles. In general, there are three distinct regions where energetic particles can be found. First, there are planetary bow shocks. Second, there are inner magnetospheres where particles can be stably trapped in well-defined radiation belts. Finally, there are the dynamic regions of the outer magnetospheres, magneto-sheaths, and magnetotails that are buffeted by the external force of the varying solar wind and the magnetic structures it contained therein." There are two distinct classes of SEP events, namely, 
"impulsive" and "gradual" (or long duration), which correspond to two physical mechanisms of particle acceleration $[2,5-11]$.

In view of the above, we study properties of SEP effective CMEs which occurred during solar cyclevspace-2pt

\section{Data}

During our study period total 93 SEP events are identified by NOAA Space Environment Services Center out of which 82 SEP events are associated with CMEs during the period 1997-2006. The data for CMEs have been taken from the catalogue maintained by the Centre for Solar Physics and Space Weather (CSPSW) (http://cdaw.gsfc.nasa.gov/CME_ list). Since LASCO/SOHO images the corona continuously since 1996 covering a field of view in the range 1.5-32 $\mathrm{R}_{\mathrm{S}}$, it represents a unique data coverage from solar minimum to maximum and beyond with a single spacecraft [12]. The SEP events are correlated with CMEs which occur within \pm 1 hour time window.

\section{Some Properties of SEP Effective CMEs}

In this section we have studied different statistical properties of SEP effective CMEs, in particular, speed, width, latitude, acceleration, mass, energy, and occurrence rate. The details are given below.

3.1. Speed of SEP Effective CMEs. Mass motion is the basic characteristic of CMEs which is quantified by their speeds. Coronagraphs obtain images with a preset time cadence. Thus, when a CME occurs, the leading edge moves to a greater heliocentric distance. On measuring the heliocentric distance of the leading edge of the CMEs in each LASCO image, one obtains CME height as a function of time. The CME speed is determined from both the linear and the quadratic fits to the height-time measurements. The speed of CME is usually measured by constructing a time-height diagram for the fastest moving feature of the CME front as it appears projected on the plane of the sky $[13,14]$. It is to be noted that the height-time measurements are made in the sky plane, so all the derived parameters are lower limits to the actual values.

The height-time (h-t) plots fitted to first order polynomials give an average speed within the LASCO field of view; but it may not be suitable for all CMEs. Quadratic fit to the h-t plot gives the constant acceleration which again is an approximation because the acceleration may also change with time [13]. The overall average and median speeds are $1431 \mathrm{~km} / \mathrm{s}$ and $1423 \mathrm{~km} / \mathrm{s}$, respectively, which is much larger than the average and median speed $435 \mathrm{~km} / \mathrm{s}$ and $369 \mathrm{~km} / \mathrm{s}$ [15] of all CMEs; whereas in their study, Gopalswamy et al., [16] show that the average speed of the SEP producing CMEs is $1557 \mathrm{~km} / \mathrm{s}$. The histogram of speeds is exhibited in Figure 1.

Figure 2 exhibits annual average speed of SEP effective CMEs. It varies from about $1150 \mathrm{~km} / \mathrm{sec}$ to $1800 \mathrm{~km} / \mathrm{sec}$.

3.2. Angular Width of SEP Effective CMEs. It is measured as the position angle extent in the sky plane, so only those CMEs

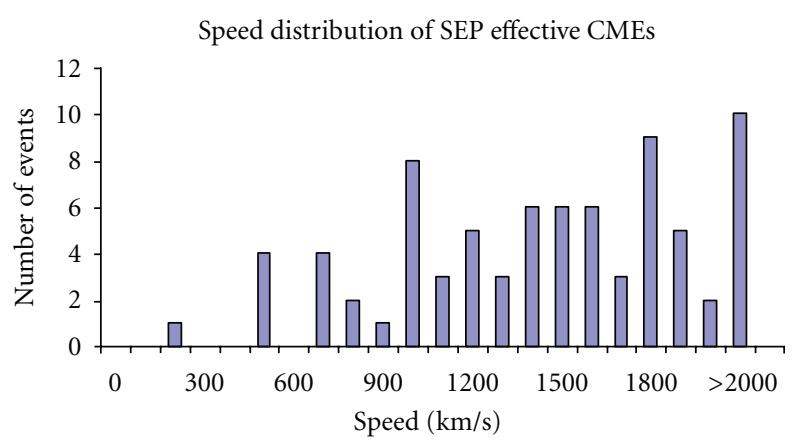

FIGURE 1: Histogram shows the speed distribution of SEP effective CMEs during 1997-2006. The numbers along $y$-axis in the figure give the number of events having a given speed.

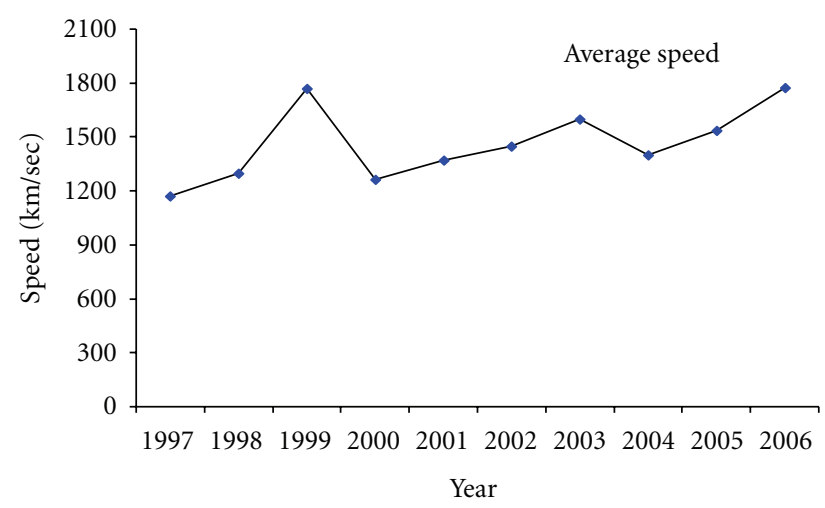

FIGURE 2: Solid line shows the annual average speed of SEP effective CMEs during 1997-2006.

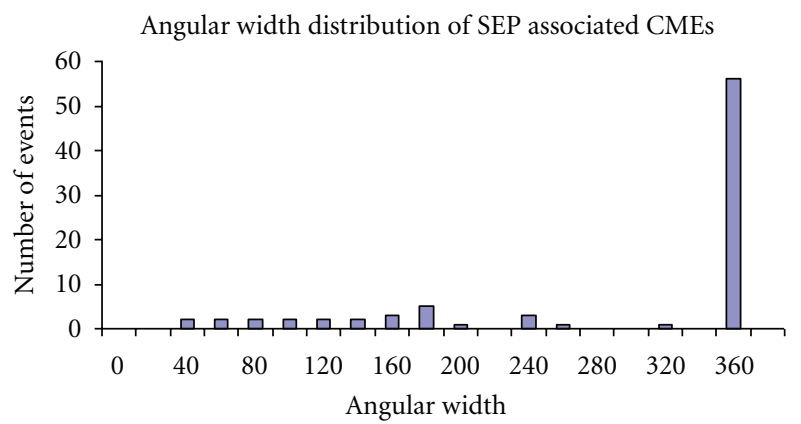

Figure 3: The width distribution of SEP effective CMEs during 1997-2006. The last bin shows angular width $360^{\circ}$, which amounts to $68 \%$ of all SEP effective CMEs. The numbers in the figure give the number of events having a given angular width.

that are in the sky plane (solar source near the limb) will yield the true width. Figure 3 is the histogram of apparent angular width of CMEs for the period 1997-2006. Clearly, the width distribution is biased towards higher CME widths.

The apparent angular width of SEP effective CMEs ranges from $30^{\circ}$ degrees to $360^{\circ}$ (cf. Figure 3 ). The average width from the 11-year-data for 82 SEP-associated CMEs is $285^{\circ}$ and the median width is $360^{\circ}$, that is, most of the SEP effective CMEs are halo in nature. The CMEs in the $360^{\circ}$ 


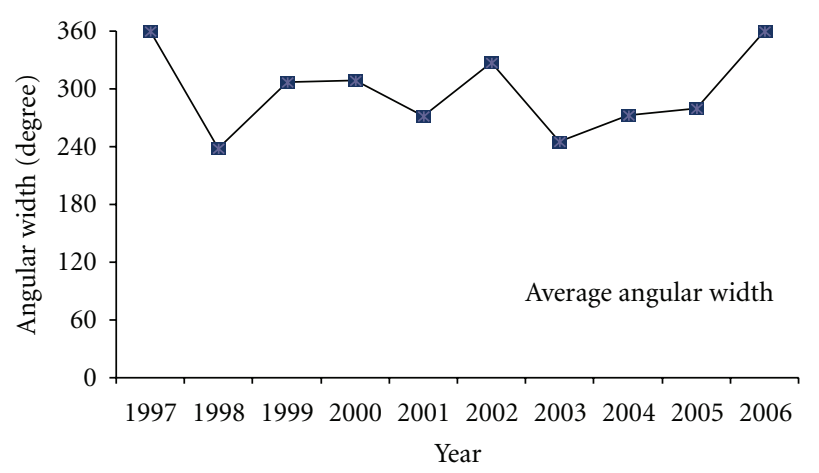

FIGURE 4: Solid line shows the annual average angular width of SEP effective CMEs during 1997-2006.

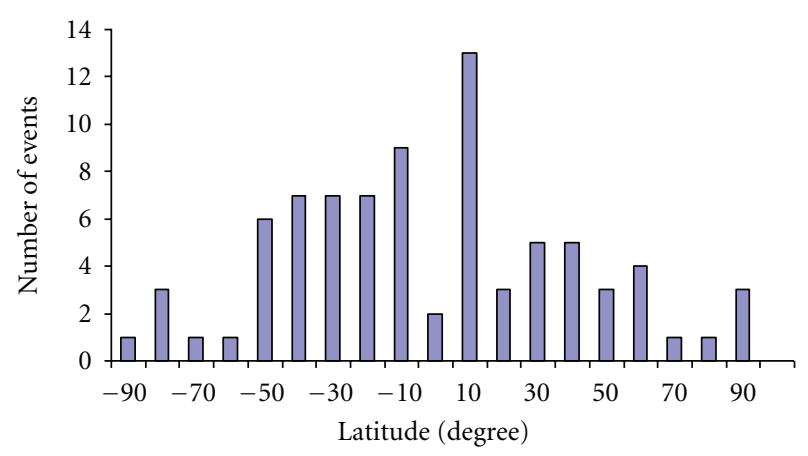

FIGURE 5: Histogram shows the latitude distribution of all SEP effective CMEs from 1997-2006. The numbers along $y$-axis in the figure give the number of events having given latitude.

bin are known as halo CMEs [17]. Halo CMEs appear to surround the occulting disk in the sky plane projection.

Figure 4 exhibits annual average angular width of SEP effective CMEs. It varies from about $235^{\circ}$ to $360^{\circ}$. Due to three-month data gap in 1998 , the value $235^{\circ}$ is unreliable.

3.3. Latitudes of SEP Effective CMEs. The CME latitude is obtained from the central position angle of the CME under the assumption that CME propagates radially away from the solar source region $[18,19]$. This assumption may not be valid during solar minimum periods when the CME path may be controlled by the global dipolar field of the Sun.

Figure 5 shows distribution of apparent latitude for the 82 SEP effective CMEs which occurred during the 10-year period (1997-2006).

It is clear from Figure 5 that most of SEP effective CMEs are ejected from southern hemisphere, although the peak number occurs at $10^{\circ}$. During the period under consideration the number of CMEs having latitudes in between $10^{\circ}$ and $-50^{\circ}$ is much larger than that in the range $>10^{\circ}$ to $-50^{\circ}$.

About $46 \%$ of all SEP effective CMEs come from northern hemisphere and about $51 \%$ come from southern hemisphere. Only $3 \%$ of CMEs come from equatorial region.

3.4. Acceleration of SEP Effective CMEs. The linear fit (constant speed) to the height-time measurements is a good

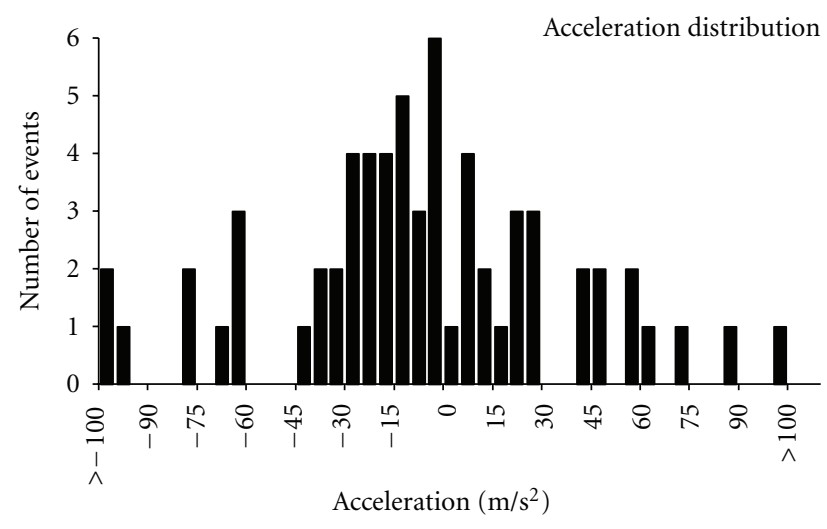

FIGURE 6: Histogram of acceleration of SEP effective CMEs, showing clear bias towards deceleration.

approximation only for some CMEs, and a second order (constant acceleration) fit is better for others. Within the coronagraphic FOV, CMEs moving faster than the slow solar wind decelerate, while the slower ones accelerate.

Figure 6 is the histogram of SEP effective CME acceleration for the period 1997-2006, that is, of cycle 23. It is clear from this figure that a majority (53\%) of CMEs are decelerated, about $17 \%$ of them move with little acceleration and the remaining 30\% have positive acceleration. Thus, CMEs have clear bias towards negative acceleration (deceleration). In all there were 64 SEP-associated CMEs whose acceleration could be determined.

All CMEs have positive acceleration in the beginning as they lift off from rest, which means the speed changes with time very early on.

From Figure 7 it is clear that there is no correlation between speed and acceleration of SEP-associated CMEs.

3.5. Mass and Energy of SEP Effective CMEs. The width is a good indicator of the mass content of CMEs. Whitelight coronagraphs detect the photospheric light scattered by the coronal electrons and therefore provide a means to measure coronal density. CMEs appear as intensity (hence, density) enhancements in a sequence of coronagraph images. The mass in a CME is estimated by determining the CME volume and the number of electrons in the CME under the assumption that the CME is fully ionized hydrogen plasma with $10 \%$ helium. Vourlidas et al. [20] explain how one could obtain the mass and energy of a CME.

Figure 8 is an 11-year (solar cycle 23) histogram involving CME mass. The average mass of 6 SEP effective CMEs; whose mass could be estimated by $\mathrm{SOHO} / \mathrm{LASCO}$ catalogue is found to be about $1.7 \times 10^{15} \mathrm{~g}$. The total mass ejected in SEP effective CMEs ranges from a few times $10^{13} \mathrm{~g}$ to more than $10^{15} \mathrm{~g}$, whereas the mass of non-SEP-associated CMEs ranges from a few times $10^{11} \mathrm{~g}$ to more than $10^{17} \mathrm{~g}$ with average value about $1.2 \times 10^{15} \mathrm{~g}$.

CMEs derive their energy from the magnetic fields in solar active regions, which contain intense magnetic fields during the solar activity maximum. Therefore, one expects more energy going into the CMEs during the maximum phase. 


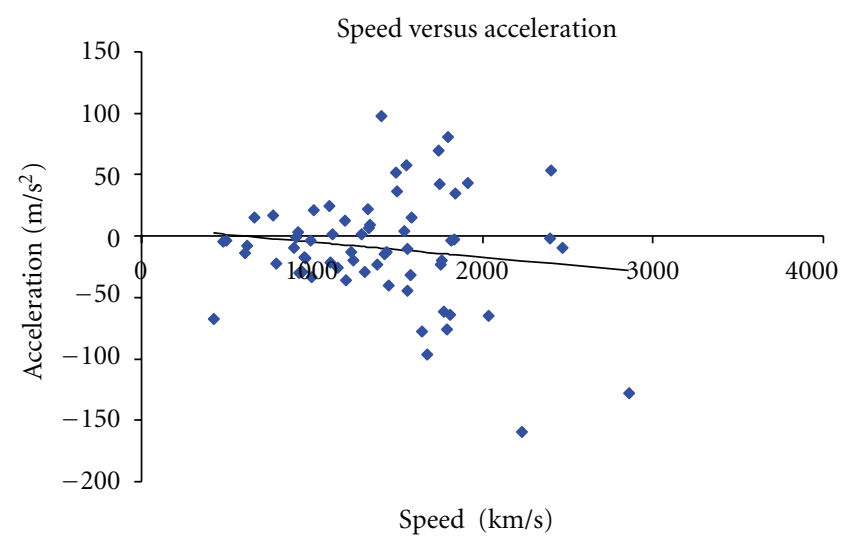

FIGURE 7: Shows no correlation between speed and acceleration as $r=-0.14$.

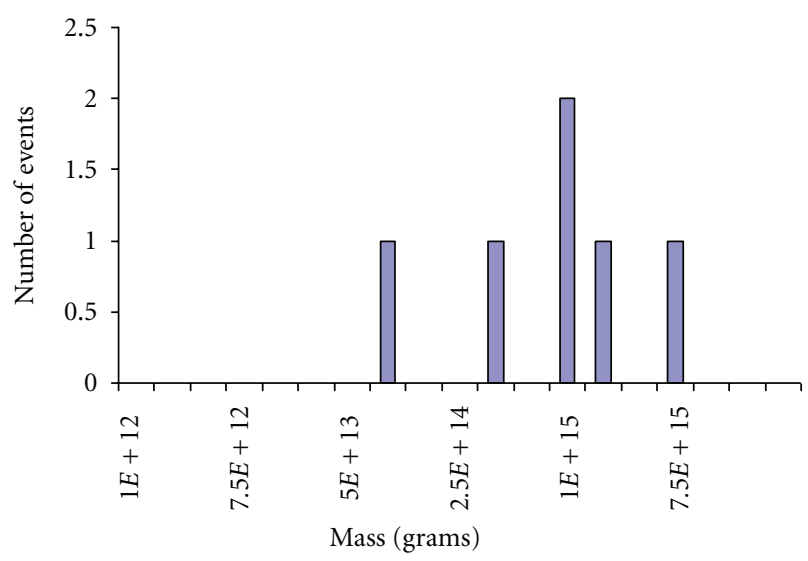

FIGURE 8: CME mass of SOHO SEP effective CMEs for the period 1997-2006.

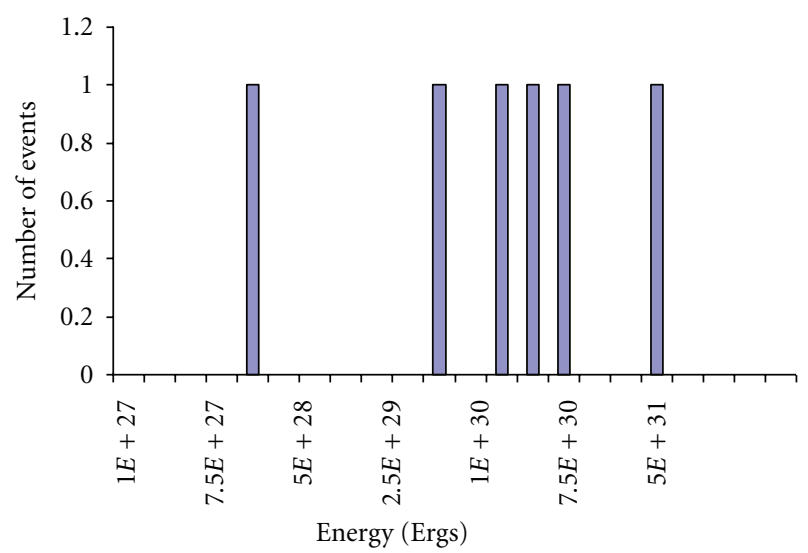

FIGURE 9: CME kinetic energy of SOHO SEP effective CMEs for the period 1997-2006.

Figure 9 is the histogram of kinetic energy of 6 SEP effective CMEs of solar cycle 23 whose energy could be estimated by $\mathrm{SOHO} / \mathrm{LASCO}$ catalogue. The kinetic energy obtained from the measured speed and mass ranges from $\sim 10^{27} \mathrm{erg}$ to $10^{31} \mathrm{erg}$ with an average value of $5.4 \times 10^{30} \mathrm{erg}$. The energy of non-SEP-associated CMEs ranges from a few

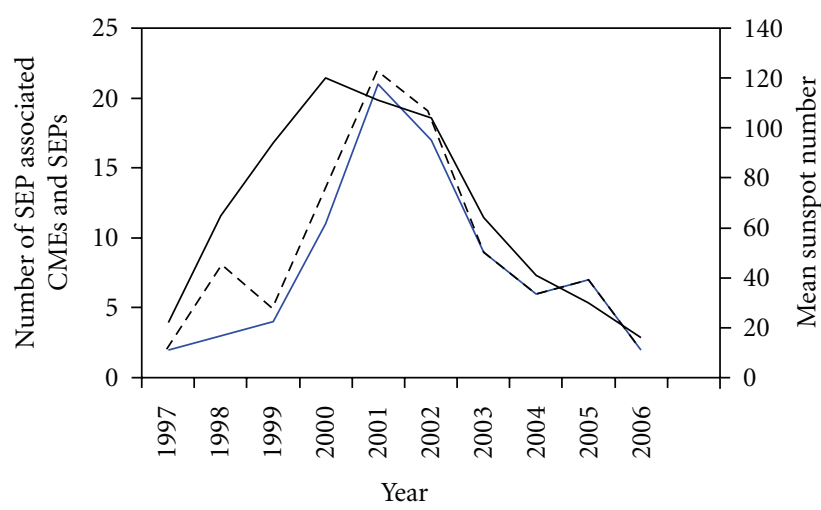

Figure 10: Occurrence rate during the period 1997-2006. Dark solid line shows annual mean sunspot numbers and blue line shows SEP effective CMEs whereas dotted line shows SEP events.

times $10^{27}$ erg to $10^{32}$ erg having average value about $1.9 \times$ $10^{30} \mathrm{ergs}$ [15]. There is large uncertainty in estimating mass and energy of remaining SEP effective CMEs.

3.6. Occurrence Rate. The Sun contributes enormously to the energetic particle population in the heliosphere through various processes: flares, CMEs, and corotating interaction regions. Here we have studied the population of SEP effective CMEs during solar cycle 23. The results are exhibited in Figure 10. It is clear from this figure that SEP effective CMEs follow the solar cycle variation, similar to sunspot numbers. In this figure, dark solid line shows sunspot number, whereas blue solid line shows SEP effective CMEs and broken line shows SEP events during solar cycle 23.

\section{Discussion and Conclusions}

The histogram of SEP effective CME speed (Figure 1) shows that there is bias towards high speeds, in contrast to non-SEP effective CMEs [15].

The histogram of apparent width (Figure 3) for all types of SEP-associated CMEs shows bias towards higher apparent angular widths $\left(360^{\circ}\right)$. That is, halo CMEs seem to be more effective for SEP events.

The CME apparent latitudes are well correlated with the latitude distribution of the helmet streamers [18], rather than with the "butterfly diagram" latitudes of active regions [21]. The histogram of latitude (Figure 5) shows a twolobe distribution with distinct peaks at midlatitudes (about $\pm 10^{\circ}$ ) in both the northern and southern hemispheres and relatively few events near the equator. This is very different to the latitude distribution of white-light CMEs, which is sharply peaked at low latitudes. Mittal and Narain [15] show that number of CMEs having latitudes in between $\pm 5^{\circ}$ and $\pm 35^{\circ}$ is much larger than those beyond $\pm 35^{\circ}$. Also Mittal et al. [22] in their study show that narrow and normal CMEs have latitude in the range $-20^{\circ}$ to $+20^{\circ}$.

The histogram of SEP effective CMEs acceleration (Figure 6) shows bias towards negative acceleration. About $53 \%$ have negative acceleration, $30 \%$ have positive acceleration, and the remaining $17 \%$ have very little acceleration. 
Non-SEP-associated CMEs are also biased towards deceleration $[14,22]$.

Figure 7 shows that there is no correlation between speed and acceleration of SEP-associated CMEs.

Vourlidas et al. [23] estimated the mass as the excess mass in the coronagraphic field of view assuming that the entire mass is located in the sky plane. Mass estimates have also been made using radio $[24,25]$ and X-ray observations [26-29]. The radio and X-ray estimates $\left(10^{14}-10^{15} \mathrm{~g}\right)$ are generally lower than the white-light mass values. This is due to the fact that the $\mathrm{X}$-ray and radio mass estimates correspond to the regions close to the Sun (and involve thermal emission properties of CME plasma), whereas the white light estimates correspond to larger heights (and involve Thomson scattering).

The histogram of mass of SEP effective CME mass (Figure 8) shows that mass is not an important parameter.

The histogram of CME kinetic energy (Figure 9) is similar to that of mass.

Flares may also produce SEP effective events.

The matter presented above leads us to conclude the following:

(1) the average and median of SEP effective CME speeds are $1431 \mathrm{~km} / \mathrm{s}$ and $1423 \mathrm{~km} / \mathrm{s}$, respectively;

(2) the average apparent width for 82 SEP effective CMEs studied by us is about $285^{\circ}$ that means most of the SEP effective CMEs are nearly halo in nature;

(3) the distribution of SEP effective CMEs is biased towards deceleration, similar to normal ones;

(4) the mass and kinetic energy of only 6 SEP effective CMEs could be determined, the average being $1.2 \times$ $10^{15} \mathrm{~g}$ and $1.91 \times 10^{30}$ ergs, respectively;

(5) about $46 \%$ of all SEP effective CMEs come from northern hemisphere and about 51\% come from southern hemisphere. Only 3\% of CMEs come from equatorial region;

(6) SEP effective CMEs follow the solar cycle variation.

\section{Acknowledgments}

The authors are thankful to FIT, Meerut, SDGI, Ghaziabad, and Meerut College, Meerut authorities for their help and encouragement. They are also thankful to IUCAA, Pune, HRI, Allahabad and ARIES, Nainital library for some relevant literature. They are highly grateful to N. Gopalswamy for many helpful correspondences. SOHO is a project of international cooperation between ESA and NASA. The authors would like to thank LASCO-CME catalogue the contributors, from which the data have been taken. They are also thankful to NOAA Space Environment Services Center for providing SEPs data.

\section{References}

[1] S. E. Forbush, "Three unusual cosmic-ray increases possiblydue to charged particles from the sun," Physical Review, vol. 70, no. 9-10, pp. 771-772, 1946.
[2] D. V. Reames, "Particle acceleration at the sun and in the heliosphere," Space Science Reviews, vol. 90, no. 3-4, pp. 413-491, 1999.

[3] K. G. McCracken, G. A. M. Dreschhoff, D. F. Smart, and M. A. Shea, "Solar cosmic ray events for the period 1561-19942. The Gleissberg periodicity," Journal of Geophysical Research A, vol. 106, no. 10, pp. 21599-21609, 2001.

[4] R. C. Carrington, "Description of a Singular Appearance seen in the Sun on September 1, 1859," Monthly Notices of the Royal Astronomical Society, vol. 20, pp. 13-15, 1859.

[5] D. V. Reames, "Energetic particles from impulsive solar flares," Astrophysical Journal, Supplement Series, vol. 73, no. 2, pp. 235-251, 1990.

[6] D. V. Reames, "Solar energetic particles: a paradigm shift," Reviews of Geophysics, vol. 33, no. 1, pp. 585-589, 1995.

[7] D. V. Reames, "Magnetic Topology of Impulsive and Gradual Solar Energetic Particle Events," The Astrophysical Journal, vol. 571, no. 1, p. L63, 2002.

[8] S. W. Kahler, "Solar flares and coronal mass ejections," Annual Review of Astronomy and Astrophysics, vol. 30, no. 1, pp. 113141, 1992.

[9] S. W. Kahler, "The correlation between solar energetic particle peak intensities and speeds of coronal mass ejections: effects of ambient particle intensities and energy spectra," Journal of Geophysical Research A, vol. 106, no. 10, pp. 20947-20955, 2001.

[10] J. T. Gosling, “The solar flare myth,” Journal of Geophysical Research, vol. 98, no. A11, pp. 18937 -18949, 1993.

[11] A. J. Tylka, "New insights on solar energetic particles from Wind and ACE," Journal of Geophysical Research A, vol. 106, no. 11, pp. 25333-25352, 2001.

[12] G. E. Brueckner, R. A. Howard, M. J. Koomen et al., "The Large Angle Spectroscopic Coronagraph (LASCO)—visible light coronal imaging and spectroscopy," Solar Physics, vol. 162, no. 1-2, pp. 357-402, 1995.

[13] N. Gopalswamy, "Coronal mass ejections of solar cycle 23," Journal of Astrophysics and Astronomy, vol. 27, no. 2-3, pp. 243-254, 2006.

[14] N. Mittal, J. Sharma, V. Tomar, and U. Narain, "On distribution of CMEs speed in solar cycle 23," Planetary and Space Science, vol. 57, no. 1, pp. 53-57, 2009.

[15] N. Mittal and U. Narain, "On some properties of coronal mass ejections in solar cycle 23," New Astronomy, vol. 14, no. 3, pp. 341-346, 2009.

[16] N. Gopalswamy, S. Akiyama, S. Yashiro, and P. Mäkelä, "Coronal mass ejections from sunspot and non-sunspot regions," in Magnetic Coupling Between the Interior and the Atmosphere of the Sun, S. S. Hasan and R. J. Rutten, Eds., Astrophysics and Space Science Proceedings, part 2, pp. 289-307, Springer, Berlin, Germany, 2010.

[17] R. A. Howard, D. J. Michels, N. R. Sheeley Jr., and M. J. Koomen, "The observation of a coronal transient directed at earth," The Astrophysical Journal, vol. 263, pp. L101-L104, 1982.

[18] A. J. Hundhausen, "Sizes and locations of coronal mass ejections: SMM observations from 1980 and 1984-1989," Journal of Geophysical Research, vol. 98, no. A8, pp. 13177-13200, 1993.

[19] N. Gopalswamy, M. Shimojo, W. Lu, S. Yashiro, K. Shibasaki, and R. A. Howard, "Prominence eruptions and coronal mass ejection: a statistical study using microwave observations," Astrophysical Journal, vol. 586, no. 1, pp. 562-578, 2003. 
[20] A. Vourlidas, P. Subramanian, K. P. Dere, and R. A. Howard, "Large-angle spectrometric coronagraph measurements of the energetics of coronal mass ejections," Astrophysical Journal, vol. 534, no. 1, p. 456, 2000.

[21] H. S. Hudson, J. L. Bougeret, and J. Burkepile, "Coronal mass ejections: overview of observations," Space Science Reviews, vol. 123, no. 1-3, pp. 13-30, 2006.

[22] N. Mittal, K. Pandey, U. Narain, and S. S. Sharma, "On properties of narrow CMEs observed with SOHO/LASCO," Astrophysics and Space Science, vol. 323, no. 2, pp. 135-145, 2009.

[23] A. Vourlidas, D. Buzasi, R. A. Howard, and E. Esfandiari, "Mass and energy properties of LASCO CMEs," in Proceedings of the The 10th European Solar Physics Meeting on Solar Variability, A. Wilson, Ed., vol. 1, pp. 91-94, ESA, Prague, Czech Republic, 2002.

[24] N. Gopalswamy and M. R. Kundu, "Thermal and nonthermal emissions during a coronal mass ejection," Solar Physics, vol. 143, no. 2, pp. 327-343, 1993.

[25] R. Ramesh, C. Kathiravan, and C. V. Sastry, "Metric radio observations of the evolution of a "halo" coronal mass ejection close to the sun," Astrophysical Journal, vol. 591, no. 2, pp. L163-L166, 2003.

[26] D. M. Rust and E. Hildner, "Expansion of an X-ray coronal arch into the outer corona," Solar Physics, vol. 48, no. 2, pp. 381-387, 1976.

[27] H. S. Hudson, L. W. Acton, and S. L. Freeland, "A long-duration solar flare with mass ejection and global consequences," Astrophysical Journal, vol. 470, no. 1, pp. 629-635, 1996.

[28] A. C. Sterling and H. S. Hudson, "Yohkoh SXT observations of X-ray "dimming" associated with a halo coronal mass ejection," Astrophysical Journal, vol. 491, no. 1, pp. L55-L58, 1997.

[29] N. Gopalswamy and Y. Hanaoka, "Coronal dimming associated with a giant prominence eruption," Astrophysical Journal, vol. 498, no. 2, pp. L179-L182, 1998. 

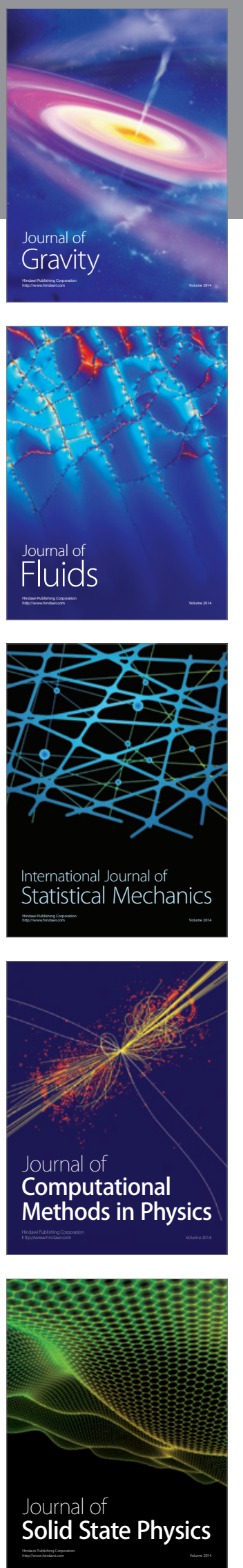

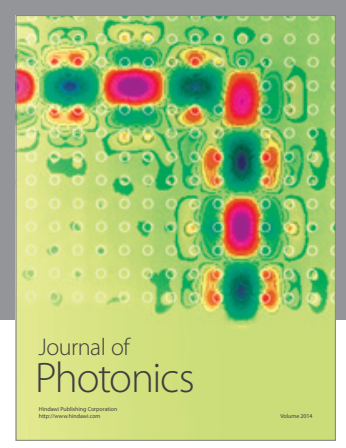

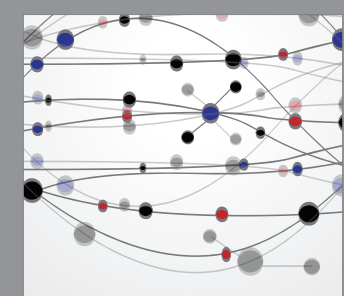

The Scientific World Journal
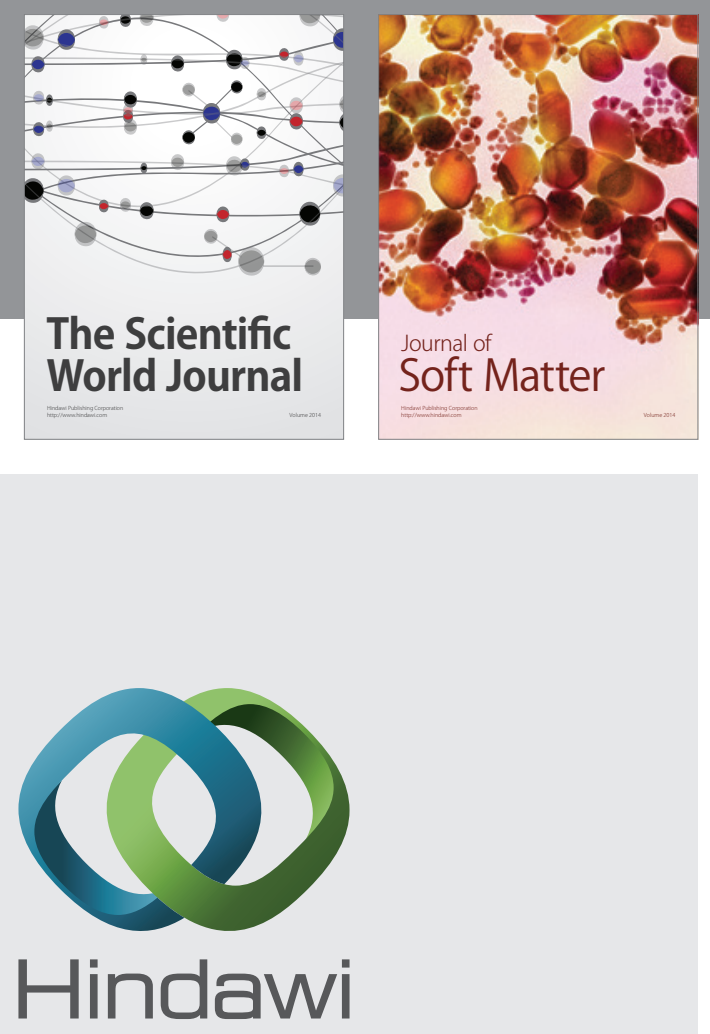

Submit your manuscripts at

http://www.hindawi.com
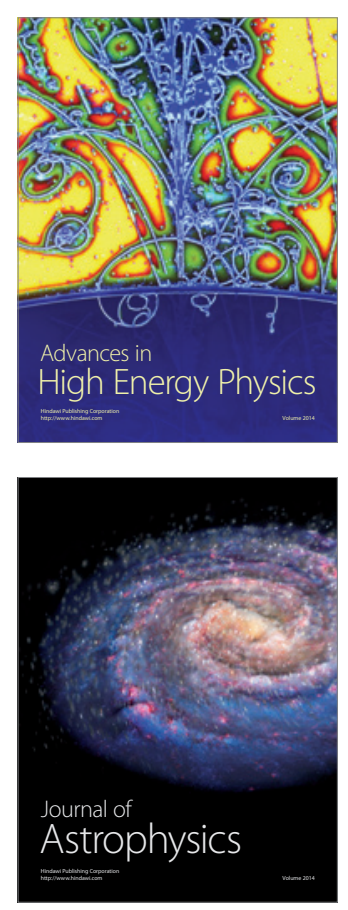
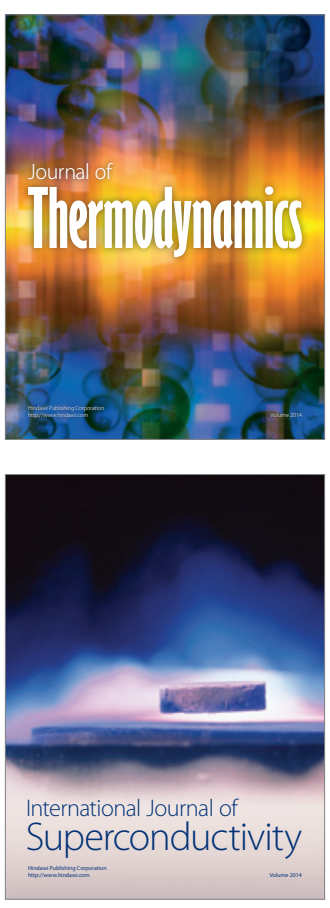
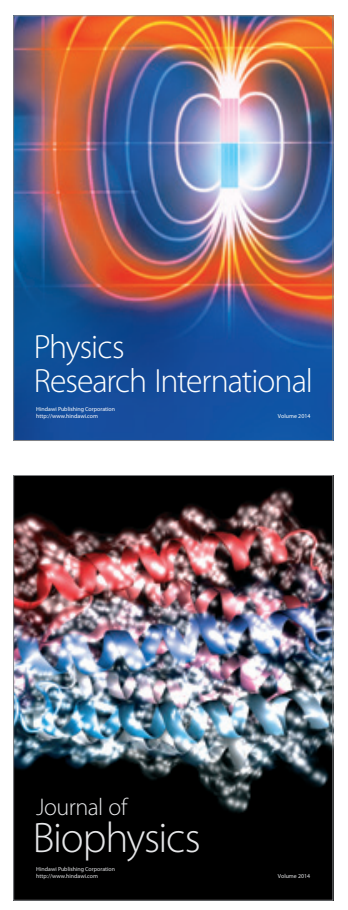
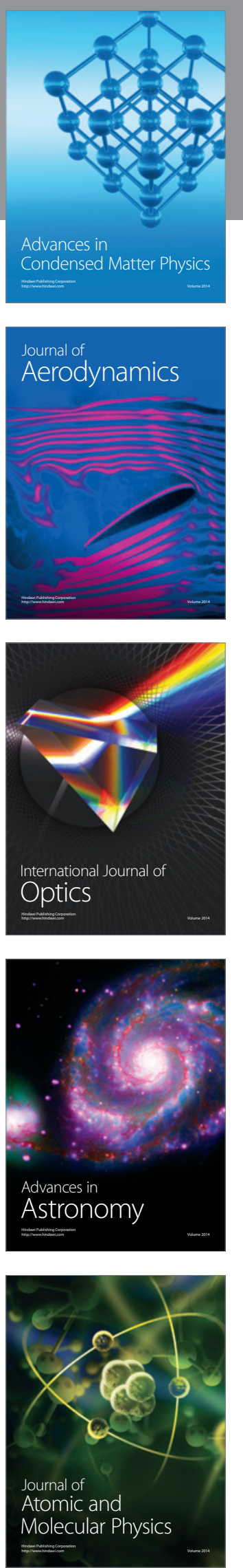